\title{
Transient Anomalous Diffusion of Telomeres in the Nucleus of Mammalian Cells
}

\author{
I. Bronstein, ${ }^{1}$ Y. Israel, ${ }^{1}$ E. Kepten, ${ }^{1}$ S. Mai, ${ }^{2}$ Y. Shav-Tal, ${ }^{3}$ E. Barkai, ${ }^{1}$ and Y. Garini ${ }^{1,4}$ \\ ${ }^{1}$ Physics Department \& Institute for Nanotechnology, Bar-Ilan University, Ramat-Gan 52900, Israel \\ ${ }^{2}$ Manitoba Institute of Cell Biology, CancerCare Manitoba, University of Manitoba, Winnipeg, Manitoba R3E OV9, Canada \\ ${ }^{3}$ The Mina \& Everard Goodman Faculty of Life Sciences \& Institute of Nanotechnology, Bar-Ilan University, Ramat-Gan 52900, Israel \\ ${ }^{4}$ Department of Imaging Science and Technology, Delft University of Technology, Lorentzweg 1, 2628 CJ Delft, The Netherlands
} (Received 10 January 2009; published 2 July 2009)

\begin{abstract}
We measured individual trajectories of fluorescently labeled telomeres in the nucleus of eukaryotic cells in the time range of $10^{-2}-10^{4} \mathrm{sec}$ by combining a few acquisition methods. At short times the motion is subdiffusive with $\left\langle r^{2}\right\rangle \sim t^{\alpha}$ and it changes to normal diffusion at longer times. The short times diffusion may be explained by the reptation model and the transient diffusion is consistent with a model of telomeres that are subject to a local binding mechanism with a wide but finite distribution of waiting times. These findings have important biological implications with respect to the genome organization in the nucleus.
\end{abstract}

DOI: 10.1103/PhysRevLett.103.018102

PACS numbers: $87.16 . Z g$, 05.40.Jc, $87.15 . \mathrm{Vv}$

The nucleus of the eukaryotic cell contains tens of thousands of genes ( 23000 in human) organized as chromosomal DNA. This crowded environment contains packed genetic material, RNA transcripts, protein factors, and a variety of nuclear bodies. The genetic information (DNA) can be either replicated to form daughter cells, or transcribed to RNA molecules leading to protein translation. These processes depend on the ability of protein factors to locate and interact with specific DNA sequence within this packed nucleus [1], as well as on the organization and structure of chromatin in the nucleus [2]. Telomeres are the end caps of the linear eukaryotic chromosomes. They play an important role in maintaining chromosome organization and integrity throughout the cell cycle. The telomeres are protected by a number of protein factors that are collectively referred to as shelterin and can bind to either the nuclear envelope, nuclear matrix, or heterochromatin, depending on the cell species [3]. Therefore, studying the dynamics of telomeres can shed light on chromosome dynamics, the role of telomeres in genome organization, and the coordination of physical structures and biological processes in the nucleus [4].

Chromosomes occupy specific nuclear volumes referred to as chromosome territories [5], and their motion is highly constrained. The diffusion of telomeres was previously studied on a limited time scale of either minutes [6] or 1-200 sec [7] and exhibited mainly normal constrained diffusion with a heterogeneous diffusion coefficient of $2-6 \times 10^{-4} \mu \mathrm{m}^{2} / \mathrm{s}$. This is significantly lower than the diffusion of small molecules such as dextran in the nucleus $\left(10-100 \mu \mathrm{m}^{2} / \mathrm{s}\right)$, which reflects the dense nature of the nucleus. The dynamics of other nuclear bodies as well as messenger RNAs were also measured [8-10] and anomalous diffusion was found for specific DNA loci [11].

In this study, we examined the diffusion properties of telomeres in the nucleus in a broad time range of almost 6 orders of magnitude $\left(10^{-2}-10^{4} \mathrm{sec}\right)$. Such a broad time range was employed by combining two different imaging setups on the same microscope. We find that the diffusion is anomalous at short times of $\sim 10^{-2}-10^{3} \mathrm{sec}$. It changes to normal diffusion at longer time intervals and the diffusion constants are found to have a wide distribution. The experiments were performed in living human cells (U2OS osteosarcoma cell line); see details in Ref. [12]. Telomeres were labeled through the shelterin subunit TRF2 fused to a green fluorescent protein. TRF2 recognizes the telomeres by attaching to the human telomeric sequences TTAGGG [6]. The fused protein was transiently expressed in the cells, and typically about 60 telomeres were observed in each cell [Fig. 1(a)] [6].
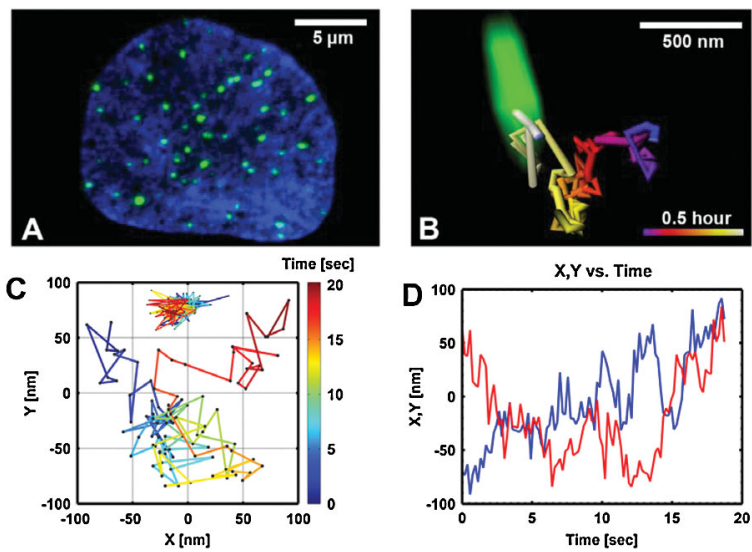

FIG. 1 (color). (A) Projection image of a 3D data. Typical distribution of telomeres (bright spots) in the nucleus stained with 4'-6-diamidino-2-phenylindole dihydrochloride (DAPI). (B) 3D motion of a telomere (bright object) as measured over $2 \times 10^{3} \mathrm{sec}$. (C) $2 \mathrm{D}$ motion of a telomere in $18 \mathrm{sec}$ measured at $5 \mathrm{~Hz}$. A fixed telomere from a dead cell is also shown (shifted upwards) indicating that the measurement precision is better than $15 \mathrm{~nm}$. (D) $X, Y$ motion of a telomere during 20 seconds measured with the confocal setup. 
In order to allow the tracking in a broad time range, we combined a CCD camera and a confocal setup to the same microscope. Confocal imaging was used for three dimensional (3D) measurements for up to 3 hours as well as two dimensional (2D) measurements at $5 \mathrm{~Hz}$ frame rate for $100 \mathrm{sec}$. The CCD was used for $2 \mathrm{D}$ fast acquisitions in a time range of $10^{-2}-10^{2} \mathrm{sec}[12]$. Almost no bleaching of the fluorescence was observed during the measurements and the signal shows fast recovery after photobleaching [12]. The images were analyzed with single particle tracking algorithms followed by mean square displacement (MSD) and further analysis [12]. In order to compare the data that were partially measured in $3 \mathrm{D}$ and partially in 2D, the MSD curves were extracted only for the 2D motion; i.e., 3D images were projected on a 2D plane.

The MSD is calculated for each telomere by finding the average displacement between each two time points with a time interval $\Delta=n \tau$ where $\tau$ is the measurement time interval and $n$ is an integer. Averaging is performed over the whole measured time,

$$
\left\langle r^{2}(\Delta)\right\rangle=\frac{1}{N-n} \sum_{m=1}^{N-n}[\mathbf{r}((m-1) \tau+\Delta)-\mathbf{r}((m-1) \tau)]^{2},
$$

where $\mathbf{r}$ is the position vector of the particle at each time point and $N$ is the total number of measured points. For normal diffusion, the MSD should follow linear dependence with $\Delta,\left\langle r^{2}(\Delta)\right\rangle=2 n_{d} D \Delta$ where $n_{d}$ is the spatial dimension and $D$ is the diffusion coefficient.

Figure 1 shows a projection of a 3D image of a nucleus [1(a)], a 3D path of a single telomere [1(b)], a typical 2D time path of a telomere [1(c)] and the $x$ and $y$ motion of a telomere [1(d)]. Figure 1(c) also shows the path of a telomere from a fixed (dead) cell. Since such a telomere is static, the observed motion indicates that the measurement precision is $\sim 15 \mathrm{~nm}$. Each telomere was localized within a limited volume of $\sim 0.04 \mu \mathrm{m}^{3}$ in a time range of $35 \min [12]$.

In contrast to previous studies, we find complex telomere dynamics never reported before. At short time scales of $10^{-2}-10^{2} \mathrm{sec}$, the diffusion is anomalous (subdiffusive) and the MSD can be expressed as $\left\langle r^{2}(t)\right\rangle=A t^{\alpha}$ with $\alpha<1$ [13]. The MSD changes to normal diffusion with $\alpha \sim 1$ at the time range of $10^{2}-10^{4} \mathrm{sec}$. In addition, the single-telomere MSD's have a very large distribution. This information sheds light on the diffusion properties in the nucleus and on the genome organization as a whole, as explained below. A transition from anomalous to normal diffusion at a crossover time $T_{\mathrm{CR}}$ was previously observed in the plasma membrane [14] and for other nuclear bodies [9], but thus far not for telomeres. Transient anomalous diffusion can be explained by the existence of binding or obstruction [15] with a finite average escape-time probability distribution. For treating transient diffusion, one can define a time-dependent diffusion coefficient: $D(t)=$ $\left\langle r^{2}(t)\right\rangle / t=A t^{\alpha-1}$ [15]. It is convenient to realize this by plotting $\left\langle r^{2}(t)\right\rangle / t$ as a function of $t$ on a log-log scale. A negative slope reflects subdiffusion (slope equal to $\alpha-1$ ) and normal diffusion is reflected as a horizontal line.

Figure 2 shows the experimental results of $\left\langle r^{2}(t)\right\rangle / t$ as a function of time $t$ for almost 6 orders of magnitude. The diffusion changes from anomalous to normal at approximately $T_{\mathrm{CR}} \approx 100 \mathrm{sec}$. The transition is not abrupt and analyzed in three different regions (Table I). The data shown emphasizes the importance of performing the particle tracking experiments at a broad time range. To verify the validity of the experimental method, the diffusion of fluorescent beads in solution was measured in the same time range. The beads were found to have a normal diffusion all across the time range [12]. The values of the coefficient $A$ in the subdiffusive time range and the diffusion coefficient $D$ in the normal diffusion time range vary considerably from one telomere to another (Fig. 3 and Table I). In contrast to that, the exponent $\alpha$ changes much less (standard deviation/mean $<0.4$ ). The value found for $\alpha$ in the range $10^{-2}-1 \sec$ (Fig. 2) is $\alpha=$ $0.32 \pm 0.12$. The data does not fit well with other models [12].

The large variation of the diffusion coefficient that is found cannot be explained by the typical size variation of telomeres in the nucleus of 5-15 kb pairs [6]. We assume that the diffusion follows the Einstein-Smoluchowski relation and Stokes's law, $D=k_{B} T / 6 \pi \eta a$, [16] where $k_{B}$ is the Boltzmann constant, $T$ the temperature, $\eta$ the viscosity, and $a$ is the particle radius. The telomeres size can be estimated based on the worm-like chain model of DNA given by $a^{2} \approx\left\langle r^{2}\right\rangle=2 d l_{p} N$ [17] where $l_{p}$ is the persistence length $(\sim 50 \mathrm{~nm}), d$ the base-pair length $(0.34 \mathrm{~nm})$, and $N$ is the number of base pairs. It therefore gives the

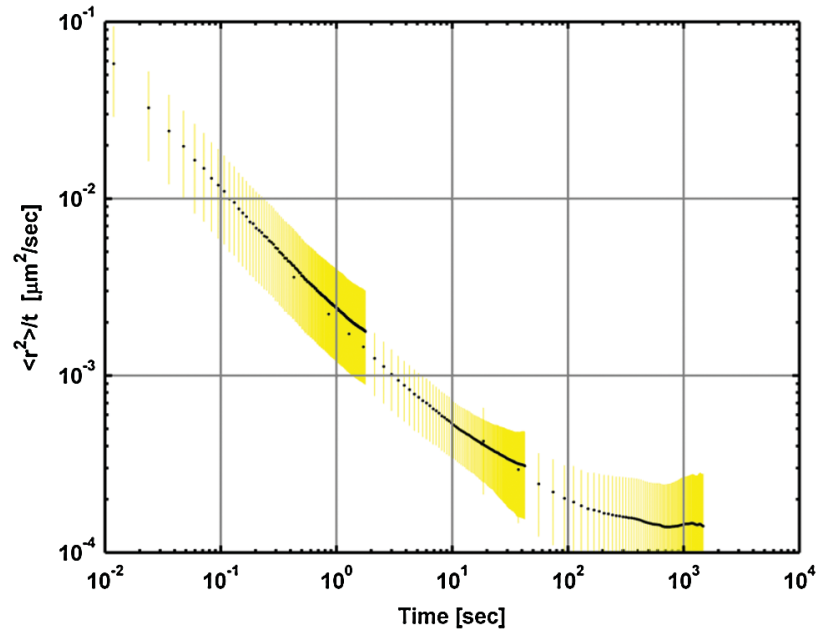

FIG. 2 (color). Log-log plot of $\left\langle r^{2}\right\rangle / t$ vs $t$. The average MSD is shown in black. The standard deviation is also shown. The data were analyzed in three time ranges (Table I). The analysis after drift and rotation correction is performed only on 2D data. The diffusion is anomalous up to $\sim 100 \mathrm{sec}$ where it changes to normal diffusion. 
TABLE I. Measured properties of the telomeres dynamics. $A$ and $\alpha$ are calculated by fitting the MSD curve to $\left\langle r^{2}(t)\right\rangle=A t^{\alpha}$. The total number of telomeres that were analyzed in each range is indicated, as well as the percentage that provided a good fit $\left(R^{2}>0.98\right)$. In each range the fit was tested with the adequate equation (either normal or anomalous). The time ranges are selected according to the curve slope.

\begin{tabular}{lllll}
\hline \hline Time range [sec] & $\alpha$ & $\mathrm{A}\left[\mu \mathrm{m}^{2} / \mathrm{sec}^{\alpha}\right]$ & $\mathrm{D}\left[\mu \mathrm{m}^{2} / \mathrm{sec}\right]$ & Number of telomeres \\
\hline $10^{-2}-10^{0}$ & $0.32 \pm 0.12$ & $(2.5 \pm 1.6) \times 10^{-3}$ & & $419(76 \%)$ \\
$10^{0}-2 \times 10^{2}$ & $0.51 \pm 0.20$ & $(1.8 \pm 1.1) \times 10^{-3}$ & & $151(47 \%)$ \\
$3 \times 10^{2}-10^{4}$ & $1.15 \pm 0.44$ & & $(5.6-5.0) \times 10^{-5}$ & $311(47 \%)$ \\
\hline \hline
\end{tabular}

extreme ratio for the telomeres as $D_{\max } / D_{\min }=$ $\left(N_{\max } / N_{\min }\right)^{1 / 2} \approx 1.73$. This is much smaller than the $\sim 18$-fold variation in the measured values of the diffusion coefficient that we find. Variation of the viscosity in the nucleus is also expected to be relatively small $[7,8]$.

We now discuss two models to interpret our data. A relevant stochastic model for the dynamics of biological entities is the well-known continuous time random walk (CTRW) model $[18,19]$. The particle undergoes a random walk with waiting times between jump events described by the probability distribution function $\psi(t)$. If the waiting time probability distribution function has a long tail, $\psi(t) \propto t^{-(1+\alpha)}$ with $0<\alpha<1$, the diffusion is anomalous $\left\langle r^{2}(t)\right\rangle \propto t^{\alpha}$. For the measured data this means that $\psi(t) \propto$ $t^{-1.32}$. A broad distribution of waiting times is possibly related to binding [13] as telomeres can bind to chromatin or the nuclear matrix through binding proteins. Even if all these processes are described by exponential distributions, a sum of such exponentials may lead to dispersive kinetics of the CTRW type. A model related to the CTRW was suggested by Saxton to describe transient anomalous diffusion in the cell and nucleus [15] and showed that a cutoff time leads to transient character of the diffusion. There, a trap model was used where anomalous diffusion of a particle is found due to trapping events in specific location (quenched disorder) which leads to power law waiting times.

Several aspects of our data are compatible with a CTRW picture. The observed transition from anomalous to normal diffusion at $T_{\mathrm{CR}} \approx 100$ happens within the CTRW language when the waiting time distribution has a cutoff, i.e., $\psi(t) \propto t^{-(1+\alpha)} d t$ for $t<T_{\mathrm{CR}}$. Such a cutoff is expected in a biological system since it corresponds to the longest (finite) binding time found in the dynamics. The value found for $T_{\mathrm{CR}}$ is similar to the short residence times found for chromatin binding proteins [20] (2-20 sec) which can bind telomeres to chromatin or nuclear matrix. The somewhat longer time that we found may indicate that the telomere binding is mediated by more than a single protein at a time.

The most striking success of the CTRW is the prediction that time averages are not reproducible observables. Simulations and theory of CTRW $[18,19]$ shows distribution of time averages MSD which is very similar to what we find in our experiments. The CTRW theory also predicts aging effects. This means that when $\psi(t)$ is a power law, the time average MSD not only depends on the lag time, but also on the total measurement time, in complete contrast to normal diffusion. We tested traces of aging in our experimental data. We considered the data only in time scales where the diffusion is anomalous since the cutoff itself may influence the aging effects. By changing the total measurement time that is used for the MSD calculation, we found that our experimental results are not sensitive to this control parameter (Ref. [12], Sec. 4). This shows that the CTRW cannot fully explain our experimental results.

Various models of polymer dynamics also predict anomalous diffusion. The famous Rouse chain model predicts $\left\langle r^{2}(t)\right\rangle \propto t^{1 / 2}$ [21], which is faster than what we find. This is not surprising, as in the crowded environment of the nucleus, the DNA is not a freely moving polymer. The de Gennes model of reptation (tube model) explains the motion of a polymer in a crowded environment by using a Rouse chain in a tube. It predicts $\left\langle r^{2}(t)\right\rangle \propto t^{1 / 4}$ with a transition to $\left\langle r^{2}(t)\right\rangle \propto t^{1 / 2}$ and even normal diffusion at longer times [22]. The transition of the diffusion is related to the time scales where the polymer starts to interact with the tube, and finally moves to distances that are larger than its own size.

Our experimental results shows a progression from anomalous to normal diffusion (roughly from $\alpha=1 / 3$ to $\alpha=1$ ) as time progresses, which is within the predictions of the reptation model. The theory of the polymer dynamics (unlike the CTRW) does not predict ergodicity breaking

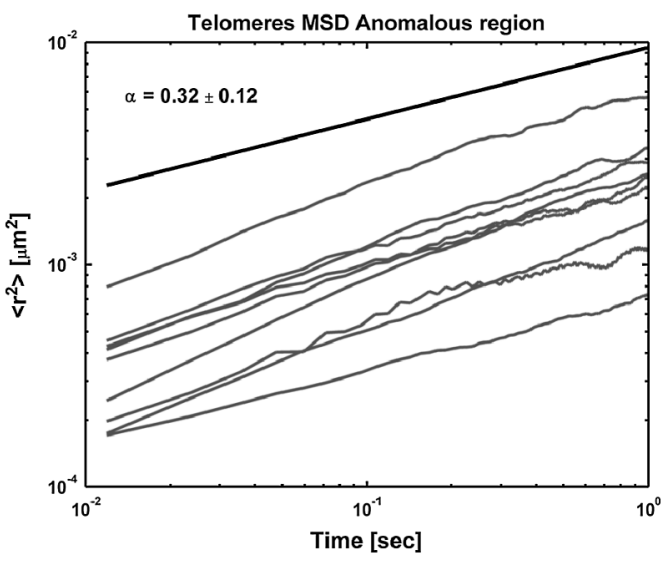

FIG. 3. The MSD of single telomeres on a log-log scale in the anomalous time range of $10^{-2}-10^{2} \mathrm{sec}$. The thick line shows the average MSD shifted upward for clarity. 
and the time averages of MSD's are reproducible observables. The large scatter of our data, i.e., the irreproducible nature of the results on the single particle level, can then be attributed to inhomogeneity. Namely, if each telomere moves in a different effective tube size (that depends on the local chromatin organization), we expect variability in the diffusion constants. In addition, the telomeres are anchored at the end of a long DNA chromatin structure, which by itself influences the telomeres dynamics. The position of the telomere with respect to its chromosome is not of major importance to the telomere diffusion because the chromosomes are tightly packed (Ref. [12], Sec. 6). Conceptually, models of polymer dynamics neglect possible binding mechanisms, but as already mentioned, the telomeres are known to have temporal binding mechanisms [3,6]. Therefore, binding effects can be important, especially in the long time limit while the short time dynamics might well be a manifestation of the de Gennes reptation model.

What is the biological benefit of slow dynamics in the cell? It was suggested previously that anomalous diffusion of a particle effects its association rate with nearby targets. A lower value of $\alpha$ increases the probability to reach nearby targets which can be favorable for transcription control in prokaryotic cells [23]. However, it is not relevant for eukaryotic cells where the transcribed messenger RNA must exit the nucleus first to be translated to a protein. On the other hand, the transient diffusion that we found indicates on a possible mechanism that maintains the genome organization in the nucleus. The chromosomes in the nucleus are localized in territories [5]. Such order ensures that nuclear organization is sustained throughout the life cycle of a cell, a property that emerges as a key contributor to genome function, though its full extent is not yet known $[2,5,24]$. It therefore raises the question on the mechanism responsible for such localization. The measured data presented here provides a possible explanation. It suggests that the short-term telomere's anomalous diffusion is governed by the telomere dynamics in its chromatin environment and that long-term diffusion is governed by telomere binding with a cutoff which altogether leads to spatial localization. Namely, anomalous subdiffusion is such a slow process that on the relevant time scale for living cells, the telomeres are nearly localized without compartments. We therefore suggest that the binding mechanism together with the reptation model which leads to transient anomalous diffusion provides a possible explanation for the upholding of genome organization.

If this phenomenon also reflects the chromosome motion in general (where chromatin sites bind to others with a limited escape time), it will lead to maintenance of structures and positions in the nucleus without the need for actual compartments. Such a mechanism has another advantage by providing flexibility. If telomere binding is switched off (e.g., by shortening the binding time), this will allow the nucleus to undergo architectural changes such as during mitosis. Indications to such mechanisms have been discussed before [3] but further studies should be performed. More specifically, it is important to measure other sites in the nucleus such as a whole chromosome and single gene loci. Measuring the effect of metabolism on the diffusion can also shed more light on the diffusion mechanisms in the nucleus.

In summary, we have measured the diffusion properties of telomeres in 6 orders of magnitude of time by combining two imaging methods. The diffusion is found to be transient from anomalous to normal. Based on these results we proposed a model for the genome organization which is important for the genomic function.

We thank Y. Rabin for valuable discussions. This work was supported by the Israel Science Foundation (ISF) and the Canadian CHIR Program "Innovative Technologies in Multidisciplinary Health Research Training."

[1] S. A. Gorski, M. Dundr, and T. Misteli, Curr. Opin. Cell Biol. 18, 284 (2006).

[2] P. Fraser and W. Bickmore, Nature (London) 447, 413 (2007).

[3] T. K. Pandita et al., Cell Mol. Life Sci. 64, 131 (2007).

[4] R. Phillips and S. R. Quake, Phys. Today 59, No. 5, 38 (2006).

[5] T. Cremer et al., Curr. Opin. Cell Biol. 18, 307 (2006).

[6] C. Molenaar et al., EMBO J. 22, 6631 (2003).

[7] X. Wang et al., Epigenetics \& Chromatin 1, 4 (2008).

[8] S. M. Görisch et al., Proc. Natl. Acad. Sci. U.S.A. 101, 13221 (2004).

[9] M. Platani et al., Nat. Cell Biol. 4, 502 (2002).

[10] Y. Shav-Tal et al., Science 304, 1797 (2004).

[11] G. G. Cabal et al., Nature (London) 441, 770 (2006).

[12] See EPAPS Document No. E-PRLTAO-103-027928 for supplementary material. For more information on EPAPS, see http://www.aip.org/pubservs/epaps.html.

[13] R. Metzler and J. Klafter, Phys. Rep. 339, 1 (2000).

[14] K. Murase et al., Biophys. J. 86, 4075 (2004).

[15] M. J. Saxton, Biophys. J. 92, 1178 (2007).

[16] H.C. Berg, Random Walks in Biology (Princeton University Press, Princeton, New Jersey, 1993).

[17] M. Rubinstein and R. H. Colby, Polymer Physics (Oxford University Press, Oxford, 2003).

[18] Y. He, S. Burov, R. Metzler, and E. Barkai, Phys. Rev. Lett. 101, 058101 (2008).

[19] A. Lubelski, I. M. Sokolov and J. Klafter, Phys. Rev. Lett. 100, 250602 (2008).

[20] R. D. Phair et al., Mol. Cell. Biol. 24, 6393 (2004).

[21] R. Shusterman, S. Alon, T. Gavrinyov, and O. Krichevsky, Phys. Rev. Lett. 92, 048303 (2004).

[22] M. Doi and S.F. Edwards, The Theory of Polymer Dynamics (Oxford University Press, Oxford, 1998).

[23] I. Golding and E. C. Cox, Phys. Rev. Lett. 96, 098102 (2006).

[24] Y. Shav-Tal, X. Darzacq, and R. H. Singer, EMBO J. 25, 3469 (2006). 